\title{
Interview with Justin Davies and Andrew Tochterman
}

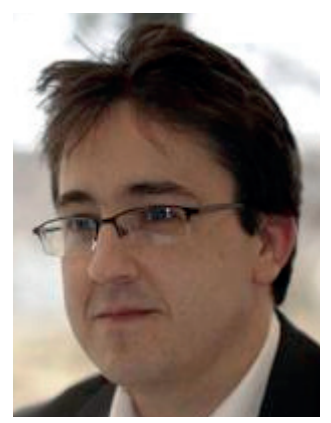

Justin Davies*,1 and Andrew Tochterman ${ }^{2}$ speak to Adam Price-Evans, Commissioning Editor of Future Cardiology: Justin Davies is a Senior Research Fellow and Honorary Consultant Cardiologist at the National Heart and Lung Institute, Imperial College London (UK). His main research interests include the development and application of pioneering technologies in engineering to aid understanding of disease mechanisms, and in the diagnosis of pathological disease states. After completing his undergraduate training at Imperial College, he was awarded a prestigious British Heart Foundation research fellowship to undertake his $\mathrm{PhD}$ at Imperial College. Since then, he has trained in interventional cardiology with a specialist interest in physiological and structural assessment of coronary disease states. In 2008, he was appointed as a Walport Clinical Lecturer to support his research activities alongside his ongoing clinical commitments.

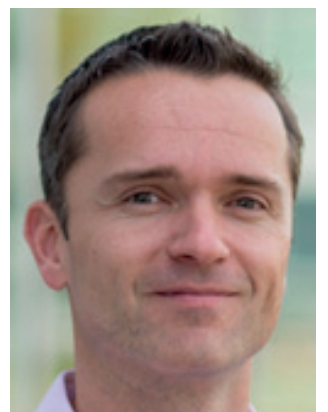

Born in Greentown, Indiana (USA), Andrew holds a BSC in Chemical Engineering from Rose-Hulman Institute of Technology (IN, USA) and an MBA from the Wharton Business School (PA, USA). His experience includes roles in Quality Engineering, R\&D, Clinical \& Product Marketing, Marketing Management and Sales at Guidant Corporation/Abbott Vascular. Andrew joined Philips Image Guided Therapy (CA, USA) in 2012, helping launch the instant wave-free ratio (iFR) product in Europe and the United States before taking on greater responsibilities as the Physiology Segment Leader, and later assuming his current role as Coronary Segment Leader at Philips Image Guided Therapy (CA, USA).

First draft submitted: 5 June 2017; Accepted for publication: 5 June 2017; Published online: 29 June 2017

\section{Q Could you both please begin by} introducing yourself, your background \& tell me a bit about your work to date? Justin: I am a Consultant Cardiologist at Imperial College London (UK) and most of my work is focused around clinical patient care in the Interventional Cardiology Laboratory and clinical research. I have been researching large artery physiology for more than 10 years. This involves many research areas, one of which is coronary physiology and physiological stenosis assessment. Most recently, we developed a new tool known as instant wave-Free Ratio (iFR) from the bench-top, taking it all the way through to commercialization and clinical trials.

'National Heart \& Lung Institute, Hammersmith Campus, Imperial College London, W12 0NN, UK

${ }^{2}$ Coronary Segment, Philips Image Guided Therapy, 3721 Valley Centre Dr \#500, San Diego, CA 92130, USA

*Author for correspondence: justin.davies@heart123.com

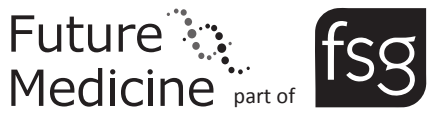


Andrew: I am the Coronary Segment Leader at Philips Image Guided Therapy and am responsible for the short-term management through to the long-term profitable growth strategy for all of our innovations in image-guided therapy devices specific to coronary artery disease. My background is an engineer by training. I started working for a Guidant Corporation and went through a series of roles in engineering, marketing, sales and now I have come in to general management here at Philips Image Guided Therapy.

Q Justin, one of your primary focuses is research in to the diagnosis \& treatment of heart disease or more specifically coronary artery stenosis. Could you tell us a bit more about coronary stenosis, its impact \& the importance of research in this area?

Justin: Coronary artery stenosis is a condition involving an abnormal narrowing in the coronary artery which causes reduction in blood flow to the heart muscle. We know these blocked arteries are often better treated with either a stent or bypass surgery however in other circumstances, they are best left alone. Differentiating between the two is important for a number of reasons such as the potentially unnecessary cost of treatment, but most importantly, these procedures are associated with both acute and long-term risks. We need to ensure that we are doing the best thing for the patient since while implanting a stent in a patient who needs it can be highly beneficial, there is no benefit and only risk for patients who don't need it. My recent research has therefore largely involved developing a tool to help distinguish which arteries should be treated with stents or bypass surgery and which arteries are best left alone.

\section{Q Fractional flow reserve (FFR) is a current} method for the assessment of coronary stenosis, however the field has begun to look toward a technique known as iFR. Could you tell us a bit about the theory behind iFR, how it works \& how the technique differs to FFR?

Justin: FFR is a pharmacological approach used for assessing the narrowing of an artery, which involves giving a powerful drug to achieve a state of constant resistance in the coronary artery and measuring the pressure drop that occurs across the stenosis. On the other hand, iFR is a more physiological approach. It uses the same wire-based technique as FFR, but, looks at the pressure drop on either side of the stenosis under rest conditions during the phase of the cardiac cycle when flow is naturally at its highest. A clear advantage of this technique is that it doesn't involve administering a drug. iFR reduces unpleasant symptoms for patients, makes assessment quicker and easier to perform and avoids any unnecessary or unwanted side effects.

Andrew: FFR is an exercise-simulated measure of ischemia however in reality, people spend the vast majority of their everyday lives under resting conditions. When a person suffers from limitations to arterial flow while at rest and present with symptoms, they will typically visit a physician or cardiologist to get an answer to their symptoms. With the correct equipment and software in place, we should be able to detect the presence of symptom-causing ischemia at rest, not just under these hyperemic or exercise-based conditions required for FFR. So in a very simplistic way, iFR is a resting measure of ischemia. From a doctor's perspective, the iFR procedure is nearly identical to that of FFR as it involves the same computers, pressure-wire technology and process to get the technology mobilized into the artery. The only major difference is that there is no need to pharmacologically stress the patient's heart to get the answer the clinician and patient are seeking.

Q Why do you think that such alternative methods have been called for \& what are the proposed benefits associated with using iFR over FFR?

Andrew: When looking back at the early days of FFR, the DEFER, FAME and FAME-2 studies truly introduced the technology and demonstrated clearly that FFR improves patient outcomes and reduces cost. Over the years, the number of patients assessed with FFR has grown as the evidence has continued to show a very consistent trend toward better patient outcomes and lower cost. Despite this evidence and despite the economic pressures on global healthcare systems, FFR technology is used in only approximately $5-8 \%$ of diagnostic characterization procedures worldwide. We therefore questioned, given the strong evidence base and the healthcare system's goal of lowering cost, why isn't this number higher? While potentially due to a number of factors, we believe a major reason is the expensive and time consuming nature of the drug administration associated with FFR. In some parts of the world, the drug is either expensive or difficult to obtain, or both, 
so this likely proposes a significant barrier to the use of this technology. We therefore believe that by presenting a more cost-effective method associated with a better patient experience and by making this information more readily available, more patients will receive a physiologybased treatment decision rather than just an anatomy-based decision.

Q Justin, you were a principal investigator on the DEFINE-FLAIR trial. Could you please tell us a bit more about this study \& its primary goals? Justin: The DEFINE-FLAIR was the largest randomized controlled trial in coronary physiology ever reported. I was the co-principal investigator on the study alongside Javier Escaned (Hospital Clinico San Carlos, Spain). This study evaluated whether using either iFR or FFR to guide revascularization therapy led to differences in hard clinical outcome, or more specifically rates of death, heart attacks or the need for a stent.

\section{Q The DEFINE-FLAIR trial actually worked in parallel with the iFR SWEDEHEART trial. Could you tell us a bit about this study \& collaboration?}

Justin: The iFR SWEDEHEART trial was carried out by a team of Scandinavian investigators led by Matthias Götberg (Lund University, Sweden) as part of the Swedish Coronary Angiography and Angioplasty Registry (SCAAR). It had an almost identical study design to the DEFINE-FLAIR trial, although was slightly smaller at around 2000 participants. Both studies looked at whether there were any differences between using iFR versus FFR for revascularization decision making. The key difference between the two is that the DEFINEFLAIR trial was a slightly larger and had a more global representation involving 50 sites in 19 countries. DEFINE-FLAIR was also a traditional randomized control trial which meant that patients were actively followed-up. The iFR SWEDEHEART trial on the other hand was entirely based in Scandinavia and it is part of their hospital record system whereby follow up was carried out by interrogating the computer to check if patients had come back in with events. It is a subtle difference between the two and in the DEFINE-FLAIR trial, we were able to perform some slightly more niche analyses that are harder to do with the registry, but they certainly complement each other very nicely. Importantly, this collaboration enabled us to have a much larger data set, when combined, than would be the case if they were separate. These studies together increased the amount of data in coronary physiology from an outcome perspective by about fourfold.

Q Results from the two trials were presented at the 2017 American College of Cardiology session in Washington DC. Could you please describe the results presented?

Justin: The results demonstrated that whether you use iFR or FFR to guide revascularization decision-making, the results are identical. There was no difference in hard clinical outcomes which is highly reassuring for patient care. Practically, this means that there is no longer any need to administer patients with drugs such as adenosine and measure FFR when you can obtain the same results using the drug-free iFR technique. iFR was also found to be quicker to perform, with a median time reduction of $4.5 \mathrm{~min}$, and a tenfold reduction in procedural side effects and symptoms. This includes a reduction in shortness of breath, chest pain, breathlessness and the need for cardioversion (an electroshock treatment required for abnormal heart murmurs caused by adenosine). Overall, the findings were neutral between iFR and FFR with regard to outcome, but were very much in favor of iFR with regards to cost and patient symptoms.

\section{Q What are the main implications of these} results \& how do you think they will impact on clinical practice \& guidelines for coronary stenosis in the future?

Andrew: We were very excited to see the most current revisions to the Appropriate Use Criteria in the US by the ACC, AHA and SCAI, which reflected that iFR has already been adapted as a suitable alternative to FFR. This is a great early endorsement of the studies we've seen thus far. To summarize in a simplistic way, these two trials have shown that whether you use an iFRguided strategy or an FFR-guided strategy for ischemia detection, you can get the same patient outcomes. However when choosing iFR, you have faster and simpler procedures, lower costs and a better patient experience. These are certainly advantages of iFR technology over FFR today and this evidence will hopefully increase use of this physiology-based detection and should result in better experiences for the patient going forward. 
Q Are there any further barriers that must be overcome before iFR is universally accepted over FFR amongst physicians?

Andrew: Certainly, having two studies, with more than 4500 total patients published in the New England Journal of Medicine provides a strong evidence base of which physicians can feel confident choosing iFR. But of course some will need more. There is a requirement for long-term evidence and so we are committed to following up the patients in these studies for 2-5 years to strengthen our evidence base. It is also important that the whole field continues to work on enhancing the guidewire technology so that consistency and performance is improved. Finally, I think that when the guidelines are revised, which normally happens approximately every 3-5 years, there will be a similar level of evidence for iFR as there is for FFR today, which will certainly help continue its adoption.

\section{Q What do you think will or should be the next stages in iFR \& coronary stenosis research?}

Andrew: We are committed to enhancing the prevalence of physiology-guided decision making relative to angiography, in terms of diagnostic catheterization. Philips Image Guided Therapy has already funded two studies that are registered on the clinicaltrial.gov website. The SAFESTEMI for Seniors trial will be looking to enhance ST-elevation myocardial infarction (STEMI) care for patients at least 65 years old with multi-vessel disease, while the MODERN study will look at how an IFR-guided strategy could cost-effectively and efficiently treat patients presenting with primary percutaneous coronary intervention (PCI) and multi vessel disease in the catheterization lab. We want to fund this research to prove that in this group of patients, physiology plays a key role in helping to improve outcomes and reduce costs.

Additionally, recently published research has suggested that approximately $15-25 \%$ of patients will still have a positive-FFR value after stent implantation based on an angiogram or imaging alone. This is another interesting area for research and it is one we are committed to studying through the DEFINE-PCI trial, which will look at using iFR to confirm that ischemia is largely resolved prior to taking the patient off the catheterization table to reduce the presentation of symptoms related to insufficient treatment.

These are a few areas where Philips is committed to evolving the field to look for new indications, enhance treatment efficiency, improve cost effectiveness and ensure get better quality patient outcomes.

Financial \& competing interests disclosure

A Tochterman is an employee of Philips Image Guided Therapy. J Davies is a consultant for Philips Image Guided Therapy, and has IP under license pertaining to the iFR technology. The authors have no other relevant affiliations or financial involvement with any organization or entity with a financial interest in or financial conflict with the subject matter or materials discussed in the manuscript apart from those disclosed.

No writing assistance was utilized in the production of this manuscript.

\section{Disclaimer}

The opinions expressed in this interview are those of the interviewees and do not necessarily reflect the views of Future Medicine Ltd. 